\title{
Um modelo estatístico para definição de áreas geográficas prioritárias para o controle do tétano neonatal
}

\author{
A statistical model for defining priority \\ geographic areas for the control of \\ neonatal tetanus
}

Joyce Mendes de Andrade Schramm 1

Célia Landmann Szwarcwald 2

\footnotetext{
1 Departamento de Epidemi ol ogia e Métodos Quantitativos, Escola Nacional de Saúde Pública, Fundação Oswaldo Cruz. Rua Leopoldo Bulhões 1480, Rio de Janeiro, RJ 21041-210, Brasil. 2 Departamento de Informações para a Saúde, Centro de Informação Científica e Tecnológica, Fundação Oswaldo Cruz. Av. Brasil 4365, Rio de Janeiro, RJ 21045-900, Brasil.
}

\begin{abstract}
A bstract We applied factor analysis to indicators of health and living conditions from Brazilian states in order to define geographic areas at potential risk for neonatal tetanus. Two factors, namely "health profile in the rural area" and "proportional mortality on neonatal tetanus" were selected and plotted against each other. A cluster composed of states from the Northeast plus Pará and Amapá was found to include most neonatal tetanus risk areas and a low case-reporting rate. Another cluster included states from the Southeast and South and displayed a neonatal tetanus reporting rate that was compati ble with that for other indicators. Espírito Santo, however, was found to be a silent productive area. The Federal District appeared alone, showing the best health conditions. Finally, the states of the MiddleWest and Roraima constituted the last cluster, characterized by intermediate health status and high neonatal tetanus case-reporting rate. Our results were consistent with the overall Brazilian health profile, distinguishing the North and Northeast from the South and Southeast, with the MiddleWest in an intermediate position.
\end{abstract}

Key words Tetanus; Newborn Infant; Factor Analysis; Risk Zones; Epi demi ology

Resumo Trata-se de um modelo estatístico para definir áreas geográficas potenciais de risco, visando ao controle do tétano neonatal. Utilizando-se a técnica estatística de componentes principais, selecionaram-se dois fatores: perfil de saúde na área rural e mortalidade proporcional por tétano neonatal respectivamente. O plot dos escores dos estados no plano cartesiano mostrou a formação de três agl omerados. No primei ro local izaram- se os estados da regi ão Nordeste, bem como Pará e Amapá, na regi ão Norte, as áreas geográficas potenciais de risco. No segundo concentraram-se os estados da região Sudeste, cuja notificação foi compatível com a si tuação apontada pel os indicadores consi derados. O Estado do Espírito Santo aparece como área produtiva silenci osa e o Distrito Federal como a uni dade com as mel hores condições de saúde. Os estados da regi ão Centro-Oeste e Rorai ma compõem o tercei ro congl omerado caracterizado por uma si tuação de saú de intermediária entre o perfil urbano e rural e com el evada notificação do agravo. O model o estatístico mostrou-se consistente com o perfil de saúde brasileiro, separando as regi ões Norte e Nordeste das regiões Sudeste e Sul, e representando em situação intermediária a re gião Centro-Oeste.

Palavras-chave Tétano; Recém-nascido; Análise Fatorial; Zonas de Risco; Epi demi ologia 


\section{Introdução}

O tétano neonatal é um agravo que está intimamente relacionado com as condições sócioeconômicas. Ocorre predominantemente em áreas rurais, em populações pobres que não possuem acesso aos serviços de saúde obstétricos e pré-natal e cujos partos são realizados no domicílio. Inúmeros aspectos relacionados às práticas das parteiras e curiosas são considerados como fatores de risco, já que levam à contaminação do cordão umbilical, que pode se dar no momento do parto ou no pós-parto imediato. A literatura descreve exaustivamente os inúmeros instrumentos utilizados para cortar o cordão umbilical e as condições de assepsia inadequadas no seu tratatamento no momento do parto e no período perinatal (Al Mukhtar, 1987; Chongsuvivatwong et al., 1991; Shafiquel Islam et al., 1982; Conyer et al., 1991; Leroy \& Garenne, 1991; Meneghel, 1988; WHO, 1988).

O tétano neonatal ocorre na maior parte das vezes até o sétimo dia de vida, evoluindo rapidamente para o óbito. É considerado uma das doenças infecciosas mais subnotificadas, pois, pelas suas características epidemiológicas, ocorre, muitas vezes, antes de a criança ter sido registrada (BIP, 1991).

No Brasil, a carência de pesquisas empíricas sobre o problema não permite uma avaliação mais abrangente da subnotificação, no nível nacional. Entretanto, dada a reconhecida subenumeração de óbitos infantis no País (Giraldelli, 1978; Laurenti, 1975; I rwing \& Oliveira, 1974; Laurenti et al., 1971), imagina-se que a subnotificação de casos de tétano seja também de grande magnitude.

Algumas investigações, realizadas em áreas específicas, demonstraram níveis muito elevados de subnotificação. Um estudo realizado pela Secretaria Estadual do Ceará estimou um sub-registro de $90 \%$ de casos para o Estado (SES, 1991). Meneghel et al. (1988), ao realizarem um inquérito epidemiológico no Município de Nonoai, Rio Grande do Sul, encontraram uma subnotificação da ordem de $50 \%$.

A partir de 1990, o Ministério da Saúde levantou uma série de dados com a finalidade de elaborar uma proposta de controle do tétano neonatal. O relatório do Programa Nacional de Imunizações de 1990 (MS, 1990a) já destacou a vacinação das mulheres em idade fértil com toxóide tetânico como a estratégia a ser adotada, colocando o antígeno à disposição das mulheres na rotina dos serviços pré-natal, principalmente em áreas classificadas como de alto risco (MS, 1990b; 1990c).
Foram definidas como áreas geográficas de risco pelo Ministério da Saúde todos os municípios cujos coeficientes médios de mortalidade por tétano neonatal tivessem igualado ou superado o valor de dois desvios-padrão acima do coeficiente de mortalidade por tétano neonatal da respectiva unidade federada. Em alguns estados, como Minas Gerais, Bahia e São Paulo, devido ao grande número de municípios, e em Rondônia, Mato Grosso e Goiás, pelo elevado número de óbitos, a ocorrência de um número mínimo de óbitos no período (dois, três ou mais) foi levada também em consideração no critério de seleção dos municípios (MS, 1990c).

Todavia, a proposta elaborada pelo Ministério da Saúde de intervir em áreas geográficas segundo um modelo de maior mortalidade ou maior incidência notificada poderia estar privilegiando áreas com menor probabilidade de ocorrência da doença, no caso de subnotificação de casos e óbitos por tétano neonatal de magnitude relevante em determinadas regiões do País. Além disso, as áreas geográficas com populações pequenas poderiam não apresentar, no período, número suficiente de casos e/ ou óbitos que conduzissem à sua seleção como área de risco e, por isso, deixar de se submeter a subseqüente controle do agravo.

Neste sentido, critérios para seleção de áreas de controle e intervenção não devem levar em consideração apenas as informações sobre a doença, pois fica difícil identificar entre as áreas geográficas onde não houve notificação de casos, quais são as áreas produtivas silenciosas (áreas de subnotificação) e quais são as áreas não produtivas, regiões onde a doença de fato não ocorreu.

Os critérios para definição de áreas geográficas de risco, cujo objetivo é o controle do agravo, têm sido definidos segundo as peculiaridades de cada país. Assim, as áreas 'repetidoras de casos' e aquelas que apresentaram, mesmo que seja somente um caso, têm sido igual mente consideradas como áreas de risco (Schramm et al., 1996). Recentemente as áreas potenciais de risco também assumiram importância. Considerando variáveis que expressam as condições sociais e de saúde da população como definidoras destas áreas, este conceito passou a ser utilizado para superar as deficiências dos sistemas de mortalidade e de vigilância epidemiológica (Silveira, comunicação pessoal).

Este trabalho, utilizando indicadores referentes não só à doença, mas também de saúde, relativos às condições de vida, propõe-se a definir uma metodologia com base na qual as 
áreas potenciais de risco possam ser identificadas. O procedimento é exemplificado usandose as informações sobre os óbitos por tétano neonatal no Brasil no período de 1979-1987.

\section{Material e métodos}

A construção de uma proposta que permitisse uma possível separação das áreas geográficas produtivas silenciosas das áreas geográficas não produtivas, implicou a seleção de um grupo de indicadores valendo-se do conhecimento da história natural do tétano neonatal.

Os indicadores selecionados para a análise foram classificados em três grupos: indicadores relacionados à presença do tétano neonatal (mortalidade proporcional por tétano neonatal, proporção de população rural, cobertura vacinal de gestantes com duas doses de toxóide tetânico, proporção de partos domiciliares); indicadores relacionados com a oferta, qualidade e cobertura dos serviços de saúde (razão médico-população; razão leito-habitantes, proporção de leitos obstétricos, proporção de óbitos por causas maldefinidas nos menores de um ano e indicadores relacionados às principais causas de morte na infância (proporção de óbitos perinatais, proporção de óbitos por diarréia entre os menores de um ano, proporção de óbitos por pneumonia entre os óbitos ocorridos nos menores de um ano, proporção de óbitos por septicemia para os menores de um ano, proporção de óbitos por doenças imunizáveis entre os menores de cinco anos). As fontes de informação e as fórmulas utilizadas no cálculo dos indicadores estão apresentadas nas TabeIas 1,2 e 3.
Na análise, utilizou-se a técnica estatística de análise fatorial, através de uma análise de componentes principais, cujo processamento foi realizado pelo programa computacional Systat. Este tipo de análise, descrita em Kleinbaum et al. (1988), é utilizada geralmente para variáveis contínuas, sem que seja(m) defini$\mathrm{da}(\mathrm{s})$ entre elas aquela(s) dependente(s), e possui como finalidade principal encontrar um número menor de dimensões que retenham o máximo de variação possível do espaço original. As relações entre cada variável original e os novos fatores são medidas pelas 'cargas' nos componentes. Por se tratarem de coeficientes de correlação, as cargas variam entre - 1 e 1 e quanto maior o seu valor absoluto, maior é a representatividade da variável original no eixo composto. Para a obtenção da 'estrutura simples', foi utilizada uma rotação varimax (Green, 1978), que determina uma nova configuração da matriz de cargas. Para a seleção dos fatores, obedeceu-se ao critério de Kaiser (Green,1978), elegendo-se aqueles cujo autovalor fosse superior a um.

Como as variáveis utilizadas na análise tratavam se de razões entre variáveis al eatórias, foi utilizada a transformação raiz quadrada como estabilizadora da variância em todas as variáveis sob estudo (Fox, 1984).

\section{Resultados}

Por intermédio da análise fatorial, foram selecionados dois fatores que explicaram $53 \%$ da variância total.

Examinando-se as cargas encontradas no primeiro fator (Tabela 1), observou-se que as

\section{Tabela 1}

Apresentação dos indicadores relacionados à mortalidade por tétano neonatal.

\begin{tabular}{|c|c|c|}
\hline Indicadores & Fórmula de construção & Fontes de informação \\
\hline $\begin{array}{l}\text { Mortalidade proporcional } \\
\text { por tétano }\end{array}$ & $\begin{array}{l}\text { Razão entre o número de óbitos por tétano neonatal } \\
\text { no período } 1983-1987 \text { e o número de óbitos } \\
\text { ocorridos entre os menores de } 28 \text { dias }\end{array}$ & $\begin{array}{l}\text { Estatísticas do Registro Civil (FIBGE, 1979; 1980; 1981; } \\
\text { 1982; 1983a; 1984; 1985; 1986; 1987) }\end{array}$ \\
\hline Proporção de população rural & $\begin{array}{l}\text { Razão entre o número de habitantes em área rural } \\
\text { e o número total de habitantes da unidade geográfica }\end{array}$ & $\begin{array}{l}\text { Projeções da População Residente (FIBGE, 1988). } \\
\text { Foi utilizada a projeção para o ano de } 1983\end{array}$ \\
\hline $\begin{array}{l}\text { Cobertura vacinal mediana } \\
\text { no período }\end{array}$ & $\begin{array}{l}\text { Foram utilizadas as estimativas fornecidas pelo } \\
\text { M inistério da Saúde para o período } 1983-1987\end{array}$ & MS, 1991 \\
\hline $\begin{array}{l}\text { Proporção de partos } \\
\text { domiciliares }\end{array}$ & $\begin{array}{l}\text { Razão entre o número de nascimentos ocorridos } \\
\text { em domicílio e registrados e o número total de } \\
\text { nascimentos registrados no período } 1983-1987\end{array}$ & $\begin{array}{l}\text { Estatísticas do Registro Civil (FIBGE, 1979; 1980; 1981; } \\
\text { 1982; 1983a; 1984; 1985; 1986; 1987) }\end{array}$ \\
\hline
\end{tabular}


seguintes variáveis apresentaram grande representatividade neste eixo: proporção de população rural, proporção de partos domiciliares, razão leito-população, mortalidade infantil proporcional por causas maldefinidas, mortalidade infantil proporcional por diarréia. De acordo com os sinais das cargas, foram encontradas correlações diretas entre a proporção de população rural com proporção de partos domiciliares, mortalidade infantil proporcional por causas maldefinidas e mortalidade infantil proporcional por diarréia. Por outro lado, pô- de-se observar a presença de correlação inversa com a variável razão leito-população e mortalidade perinatal. Estes achados indicam que o primeiro componente expressa um perfil de saúde para áreas rurais, isto é, áreas com elevadas proporções de partos domiciliares, proporções de óbitos por causas maldefinidas, mortalidade infantil proporcional por diarréia e com baixa oferta de leitos hospitalares. Assim, de acordo com os resultados encontrados decidiu-se denominar este fator de Perfil de Saúde na Área Rural.

Tabela 2

Indicadores relacionados à oferta, qualidade e cobertura dos serviços de saúde.

\begin{tabular}{|c|c|c|}
\hline Indicadores & Fórmulas de construção & Fontes de informação \\
\hline Razão médico-população & $\begin{array}{l}\text { Razão entre o número de médicos existentes } \\
\text { e a população para o ano de } 1983\end{array}$ & Fibge, 1988, 1983b \\
\hline Razão leito-habitantes & $\begin{array}{l}\text { Razão entre o número total de leitos disponíveis para } \\
\text { o ano de } 1983 \text { e a população da unidade federada }\end{array}$ & Fibge, $1988 ; 1983 b$ \\
\hline Proporção de leitos obstétricos & $\begin{array}{l}\text { Razão entre o número total de leitos obstétricos e o } \\
\text { número total de leitos disponíveis para o ano de } 1983\end{array}$ & Fibge, 1983b \\
\hline $\begin{array}{l}\text { Proporção de óbitos por } \\
\text { causas maldefinidas nos } \\
\text { menores de um ano }\end{array}$ & $\begin{array}{l}\text { Razão entre o número de óbitos por causas } \\
\text { maldefinidas e o total de óbitos ocorridos nos } \\
\text { menores de um ano em } 1983\end{array}$ & MS 1987 \\
\hline
\end{tabular}

Tabela 3

Indicadores relacionados a outras causas de morte na infância.

\begin{tabular}{|c|c|c|}
\hline Indicadores & Fórmulas de construção & Fontes de informação \\
\hline $\begin{array}{l}\text { Proporção de óbitos } \\
\text { por causas perinatais }\end{array}$ & $\begin{array}{l}\text { Razão entre o número de óbitos por causas perinatais e o número de óbitos } \\
\text { ocorridos nos menores de um ano, excluindo-se os óbitos por sintomas } \\
\text { e causas maldefinidas, para o ano de } 1983\end{array}$ & MS, 1987 \\
\hline $\begin{array}{l}\text { Proporção de óbitos por } \\
\text { septicemia entre os menores } \\
\text { de um ano }\end{array}$ & $\begin{array}{l}\text { Razão entre o número de óbitos por septicemia ocorridos nos menores } \\
\text { de um ano, excluindo-se os óbitos por sintomas e causas maldefinidas, } \\
\text { para o ano de } 1983\end{array}$ & MS, 1987 \\
\hline $\begin{array}{l}\text { Proporção de óbitos por } \\
\text { diarréia entre os menores } \\
\text { de um ano }\end{array}$ & $\begin{array}{l}\text { Razão entre o número de óbitos por infecções intestinais e o total de óbitos } \\
\text { nos menores de } 1 \text { ano, excluindo-se do denominador os óbitos por sintomas } \\
\text { e causas maldefinidas, para o ano de } 1983\end{array}$ & MS, 1987 \\
\hline $\begin{array}{l}\text { Proporção de óbitos por } \\
\text { pneumonia }\end{array}$ & $\begin{array}{l}\text { Razão entre o número de óbitos por pneumonia e o total de óbitos } \\
\text { nos menores de um ano, excluindo-se do denominador os óbitos } \\
\text { por sintomas e causas maldefinidas, para o ano de } 1983\end{array}$ & MS, 1987 \\
\hline $\begin{array}{l}\text { Proporção de óbitos por } \\
\text { doenças imunizáveis nos } \\
\text { menores de cinco anos }\end{array}$ & $\begin{array}{l}\text { Razão entre o número de óbitos por causas imunizáveis e o total de óbitos } \\
\text { nos menores de cinco anos, excluindo-se do denominador os óbitos por sintomas } \\
\text { e causas maldefinidas, para o ano de } 1983\end{array}$ & MS, 1987 \\
\hline
\end{tabular}


No segundo fator, cargas grandes em valor absoluto correspondem às variáveis mortalidade proporcional por tétano neonatal, proporção de leitos obstétricos e mortalidade infantil proporcional por septicemia. Como esperado, observa-se a existência de correlação inversa entre a mortalidade neonatal proporcional por tétano e a proporção de leitos obstétricos. Adicionalmente, verifica-se uma correlação direta entre a mortalidade neonatal proporcional por tétano e a mortalidade infantil proporcional por septicemia, o que igualmente pode ser considerado como coerente, na medida em que a mortalidade infantil proporcional por septicemia foi utilizada como indicador da qualidade da assistência (Becker, 1991). Explicando predominantemente a doença, este fator foi denominado de Mortalidade Proporcional por Tétano Neonatal.

Os escores das unidades federadas no plano cartesiano formado pelos dois primeiros componentes estão representados na Figura 1. Examinando-se o gráfico no sentido do círculo trigonométrico, verifica-se que nos quadrantes I e II estão representados os estados das regiões Norte e Nordeste. No quadrante I encontram-se as unidades federadas com maior proporção de população rural e alta mortalidade por tétano neonatal. No quadrante II, estão localizados os estados com baixa predominância de população rural e alta mortalidade por tétano neonatal efinalmente, nos quadrantes III e IV, encontram-se distribuídas as unidades federadas com baixa mortalidade por tétano neonatal notificada, sendo que, no quadrante III, estão as que têm maior proporção de popuIação urbana, ao passo que, no quadrante IV, estão aquelas com predominância de população rural.

De acordo com a distribuição dos escores das unidades federadas no plano cartesiano composto pelos dois primeiros componentes, foi possível observar a formação de aglomerados das mesmas, delimitados por meio de elipses, conforme apresentado na Figura 1. No quadrante IV, identifica-se um aglomerado formado por estados da região Nordeste e os Estados do Pará e Amapá. Retornando ao objetivo inicial deste trabalho, chama-se atenção para este aglomerado: é o que contém predominantemente as áreas geográficas potenciais de risco, já que têm, por um lado, perfil de saúde compatível com a área rural e, por outro, baixa mortalidade notificada de tétano neonatal. Neste aglomerado, destaca-se o Estado da Paraíba, que, provavelmente, é uma das áreas geográficas de maior subnotificação de casos.
Tabela 4

Resultado da análise de componentes principais.

\begin{tabular}{lcc}
\hline Variáveis & Fator 1 & Fator 2 \\
\hline Mortalidade proporcional por tétano neonatal & 0,32 & 0,71 \\
Proporção de população rural & 0,84 & 0,24 \\
Proporção de partos domiciliares & 0,89 & 0,19 \\
Mediana da cobertura vacinal & 0,53 & $-0,18$ \\
Razão médico-população & $-0,09$ & 0,23 \\
Razão leito-população & $-0,77$ & 0,14 \\
Proporção de leitos obstétricos & 0,30 & $-0,71$ \\
Mortalidade proporcional por causas maldefinidas & 0,71 & $-0,15$ \\
Mortalidade perinatal & $-0,52$ & $-0,49$ \\
Mortalidade proporcional por septicemia & $-0,33$ & 0,77 \\
Mortalidade proporcional por diarréia & $-0,91$ & 0,10 \\
Mortalidade proporcional por pneumonias & $-0,55$ & $-0,39$ \\
Mortalidade proporcional por doenças imunizáveis & 0,11 & 0,04 \\
para menores de cinco anos & & \\
\% Variância total explicada & 36,00 & 17,00 \\
\hline
\end{tabular}

Figura 1

Escores padronizados nos componentes principais 1 e 2, segundo as unidades federadas.

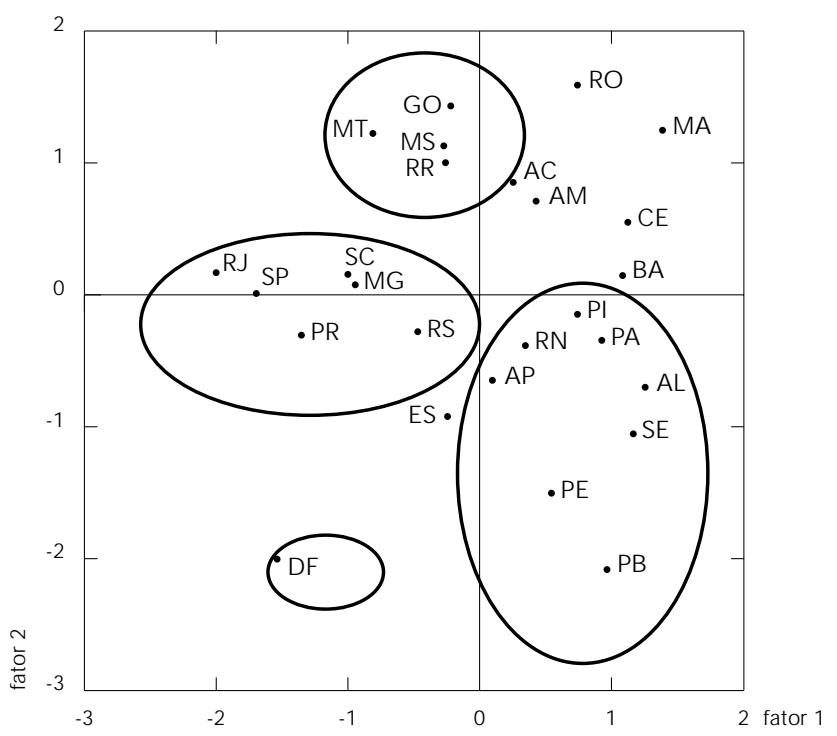

O segundo aglomerado é formado por estados da região Sudeste, que são aqueles com maior proporção de população urbana e com notificação de tétano neonatal compatível com a situação apontada por meio dos outros indicadores considerados. Entre os dois aglomerados está localizado o Estado do Espírito Santo, 
identificado, assim, como uma provável área produtiva silenciosa. No terceiro quadrante, sobressai-se, ainda, o Distrito Federal como a unidade que apresenta as melhores condições de saúde do Brasil, em situação destacada mesmo em relação aos estados que compõem o aglomerado ali localizado.

Os Estados de Goiás, Mato Grosso do Sul e Roraima formam um terceiro conglomerado caracterizado por uma situação de saúde intermediária entre o perfil urbano e rural e, sobretudo, pela el evada notificação de tétano neonatal.

\section{Considerações finais}

A análise fatorial pelo método de componentes principais mostrou-se uma técnica sensível tanto para selecionar os indicadores relacionados com o risco da ocorrência da doença, como para detectar áreas potenciais de risco.

O modelo estatístico proposto mostrou-se consistente com o perfil de saúde brasileiro, separando nitidamente o Nordeste e o Norte do Sudeste e do Sul e representando em situação intermediária os estados da região CentroOeste. Além disto, de acordo com o que seria esperado, o Distrito Federal ficou isolado das outras Unidades da Federação e foi identificado como a área geográfica de melhores condições de saúde.
Da análise estatística, ficou também destacado o fato de que a mortalidade proporcional por tétano neonatal constituiu-se em um fator independente do composto pelos indicadores que representam predominantemente o perfil de saúde na área rural. Considerando o quadro teórico que embasa a história natural da doença, estes achados demonstram a elevada subnotificação de casos de tétano neonatal em diversas áreas do território nacional. Adicionalmente, apontam para o perigo de se elaborarem critérios baseados simplesmente nos casos conhecidos pelo sistema de vigilância epidemiológica e pelo sistema de mortalidade.

Dado o grande aglomerado identificado como área produtiva silenciosa, formado por várias unidades das regiões Nordeste e Norte, evidencia-se a necessidade de se proporem modelos alternativos para a identificação de áreas de risco que não aqueles baseados apenas em taxas da doença, sobretudo no nível de município.

Para o controle da doença no Brasil, considera-se como etapa fundamental a investigação da distribuição geográfica dos casos notificados, visando identificar os municípios que não têm notificação de tétano neonatal em acordo com suas condições de saúde. Uma solução possível seria a de reproduzir o modelo estatístico proposto, porém considerando-se os municípios como unidades de análise. Neste caso, a identificação das áreas potenciais de risco poderia ser realizada por meio de técnicas de geoprocessamento e/ ou análise espacial.

\section{Referências}

AL MUKHTAR, M. Y., 1987. Birth care practice and tetanus neonatorum: a hospital-based study in Mosul. Public Health, 101:453-456.

BECKER, R. A., 1991. Análise de Mortalidade/Del ineamentos Básicos. Brasília: Centro de Documentação, Ministério da Saúde. (mimeo.)

BIP (Boletin Informativo Pai), 1991. La Mortalidad por Tétanos Neonatal. Washington: Opas (Organización Panamericana de Salud).

CHONGSUVIVATWONG, V.; BUCHARKONRN, L. \& RUTHAIPORN-TREETONG, R. N., 1991. Traditional birth attendants in an endemic area of tetanus neonatorum in Thailand: pitfalls in the control program. Journal of Community Health, 16:325-331. 
CONYER, R. T.; AMOR, J. S.; IZABA, B. S.; DIAZ, A. T.; CARRILLO, L. L.; MUÑOZ, J. L. C. \& RINCÓN, H. G., 1991. Factores associados a la mortalidad por tétanos neonatal en el área rural de Jalisco. Salud Pública de México, 33:207-213.

FIBGE (Fundação Instituto Brasileiro de Geografia e Estatística), 1979. Estatísticas do Registro Civil. Vol. 6, Rio de Janeiro: FIBGE.

FIBGE (Fundação Instituto Brasileiro de Geografia e Estatística), 1980. Estatísticas do Registro Civil. Vol. 7, Rio de Janeiro: FIBGE.

FIBGE (Fundação Instituto Brasileiro de Geografia e Estatística), 1981. Estatísticas do Registro Civil. Vol. 8, Rio de Janeiro: FIBGE.

FIBGE (Fundação Instituto Brasileiro de Geografia e Estatística), 1982. Estatísticas do Registro Civil. Vol. 9, Rio de Janeiro: FIBGE.

FIBGE (Fundação Instituto Brasileiro de Geografia e Estatística), 1983a. Estatísticas do Registro Civil. Vol. 10, Rio de Janeiro: FIBGE.

FIBGE (Fundação Instituto Brasileiro de Geografia e Estatística), 1983b. Estatísticas de Saúde/Assistência Médico-Sanitária. Vol. 8, Rio de Janeiro: FIBGE.

FIBGE (Fundação Instituto Brasileiro de Geografia e Estatística), 1984. Estatísticas do Registro Civil. Vol. 11, Rio de Janeiro: FIBGE.

FIBGE (Fundação Instituto Brasileiro de Geografia e Estatística), 1985. Estatísticas do Registro Civil. Vol. 12, Rio de Janeiro: FIBGE.

FIBGE (Fundação Instituto Brasileiro de Geografia e Estatística), 1986. Estatísticas do Registro Civil. Vol. 13, Rio de Janeiro: FIBGE.

FIBGE (Fundação Instituto Brasileiro de Geografia e Estatística),1987. Estatísticas do Registro Civil. Vol. 14, Rio de Janeiro: FIBGE.

FIBGE (Fundação Instituto Brasileiro de Geografia e Estatística), 1988. Projeções de População Residente e do Número de Domicílios Particulares Ocupados, 1985-2020. Vol. 1, Rio de Janeiro: FIBGE.

FOX, J., 1984. Linear Statistical Models and Related Methods with Applications to Social Research. New York: John Willey \& Sons.

GREEN, P. E., 1978. Analzying Multivariate Data. Illinois: The Dryden Press.

GIRALDELLI, B. W., 1978. Avaliação da Qualidade das Estatísticas de Nascimento para o Estado de São Paulo (Sub-Registro no Ano de 1985). In: Atas do 3o Simpósio Nacional de Probabilidade e Estatística, São Paulo: Universidade de São Paulo.

IRWING, R. \& OLIVEIRA, L. A. P., 1974. Tendências e possibilidades na coleta de estatísticas vitais no Brasil. Revista Brasileira deEstatística, 35:179-202.

KLEINBAUN, D. J.; KUPPER, L. L. \& MULLER, K. E., 1988. Applied Regression Analyses and other Multivariate Methods. 2a ed., Boston: PWS-Kent Publishing Company.
LAU RENTI, R.; SAYÃO, F.A. \& SILVEIRA, M . H., 1971 Sub-registro de nascimento de crianças falecidas com menos de um ano de idade. Revista de Saúde Pública, 5:237-242.

LAURENTI, R., 1975. Fatores de erros na mensuração da mortalidade infantil. Revista de Saúde Pública, 5:237-242.

LEROY, O. \& GARENNE, M., 1991. Risk factors of neonatal tetanus in Senegal. International Journal of Epidemiology, 20:521-526.

MENEGHEL, S.; DANILEVICZ, N.; KMETZSEH, C. \& VON MUHLERN, D., 1988. Inquérito de morbimortalidade do tétano neonatal no Município de Nonoai, Rio Grande do Sul, 1988. Relatório de pesquisa. Porto Alegre (mimeo.).

MENEGHEL, S. N., 1988. Vigilância epidemiológica do Tétano no Rio Grande do Sul, Brasil. Boletin de la Oficina Sanitária Panamericana, 105:139-150.

MS (Ministério da Saúde), 1987. Estatísticas de Mortalidade, Brasil, 1983. Brasília: Centro de Documentação, Ministério da Sáude.

MS (Ministério da Saúde), 1990a. Programa de Controle do Tétano Neonatal no Brasil. Uma Proposta de Ação. Brasília: Centro de Documentação, Ministério da Sáude. (mimeo.)

MS (Ministério da Saúde), 1990b. O Enfoque de Risco na Eliminação do Tétano Neonatal. Brasília: Centro de Documentação, Ministério da Sáude. (mimeo.)

MS (Ministério da Saúde), 1990c. Subsídios para Elaboração de um Plano Nacional de Controle do Té tano. Brasília: Centro de Documentação, Ministério da Sáude. (mimeo.)

MS (Ministério da Saúde), 1991. O Enfoque de Risco na Eliminação do Tétano Neonatal no Brasil. Brasília: Centro de Documentação, Ministério da Sáude. (mimeo.)

SES (Secretaria Estadual de Saúde), 1991. Programa de Eliminação do Tétano Neonatal. Fortaleza: SES.

SCHRAMM, J. M. A.; SANCHES, O. \& SZWARCWALD, C. L., 1996. Análise da mortalidade por tétano neonatal no Brasil (1979-1987). Cadernos de SaúdePública, 12:217-224.

SHAFIQUEL ISLAM, M.; MUJIBUR RAHAMAN, M.; AZIZ, K. M. S. \& PATWARI, Y., 1982. Birth care practice and neonatal tetanus in rural area of Bangladesh. Journal of Tropical Pediatrics, 28: 299-302.

WHO (World Health Organization), 1988. Expanded Programme on Immunization. Weekly Epidemiological Record, 3:10-13. 